DFUPG-53-00

\title{
On the Doubling Phenomenon in Lattice Chern-Simons Theories *
}

\author{
F. Berruto ${ }^{(a)}$, M.C. Diamantini ${ }^{\dagger(b)}$ and P. Sodano ${ }^{(a)}$ \\ a)Dipartimento di Fisica and Sezione I.N.F.N., \\ Universitá di Perugia, Via Pascoli I-06123 Perugia, Italy \\ b)CERN, Theory Division, CH-1211 Geneva, Switzerland
}

\begin{abstract}
We analyse the pure Chern-Simons theory on an Euclidean infinite lattice. We point out that, as a consequence of its symmetries, the Chern-Simons theory does not have an integrable kernel. Due to the linearity of the action in the derivatives, the situation is very similar to the one arising in the lattice formulation of fermionic theories. Doubling of bosonic degrees of freedom is removed by adding a Maxwell term with a mechanism similar to the one proposed by Wilson for the fermionic theories.
\end{abstract}

\section{Introduction}

In odd space-time dimensions, there is the possibility of adding a gauge invariant, topological Chern-Simons (CS) term to the gauge field action. The CS term breaks both the parity and time-reversal symmetries and, when coupled with a Maxwell or Yang-Mills term, leads to massive gauge excitations [1]. For an Abelian model in three space-time dimensions, the pure CS Lagrangian is defined as

$\mathcal{L}_{C S}=\frac{k}{2} A_{\mu} \epsilon^{\mu \alpha \nu} \partial_{\alpha} A_{\nu}$

where $k$ is a dimensionless coupling constant.

The pure CS theory is a topological field theory [2]. Being dominant at large distances, the CS action may be used as a low energy effective field theory for condensed matter systems [3].

While in the continuum the pure CS theory is exactly solvable, things are quite different on the lattice: in fact the kernel defining the CS action exhibits a set of zeroes which are not due to gauge invariance [4] and the theory is not integrable even after gauge fixing. The action (11) is of first order in the derivatives, and the appearence of extra zeros in its lattice formulation is reminescent of the "doubling" of fermions on the lattice [5]. Doubling phenomena for bosonic

\footnotetext{
*Presented by P. Sodano at Lattice '00, Bangalore, India. ${ }^{\dagger}$ Supported by a Swiss National Science Foundation fellowship.
}

degrees of freedom have been already evidenced in Euclidean lattice gravity [6] and in the analysis of the coupling of gravity to matter on the lattice [7].

Previous studies of pure CS theory on the lattice have been carried out using the Hamiltonian formalism in [8], by introducing a mixed CS action with two gauge fields with opposite parity or by means of two gauge fields living on the links of two dual lattices (thereby obtaining in both cases a parity even action) [9].

Here we shall evidence that as a result of the theorem proved in 10 the non-integrability of the CS kernel is a general feature of any gaugeinvariant, local, parity odd and cubic symmetric gauge theory on an infinite Euclidean lattice provided that under parity $A_{\mu}(\vec{x}) \longrightarrow A_{\mu}(-\vec{x}-\hat{\mu})$ and $A_{\mu}(\vec{p}) \longrightarrow-A_{\mu}(-\vec{p})$. Since the addition of a Maxwell term regularizes the CS action, the presence of the extra zeros in the CS action did not cause any problem in previous investigations of the Maxwell-CS action on the lattice [4 11]. Although in physical applications one is naturally lead to study the dynamics of gauge models described by a Maxwell-CS action, the proper definition of a pure CS action on the lattice is an interesting problem in its own right since it could open the way to a lattice evaluation of topological invariants [2].

The question naturally arises if it is possible to define a parity on the lattice so that the Chern- 
Simons term is parity odd but the kernel is integrable.

\section{Euclidean Chern-Simons theory on the lattice}

We consider an infinite Euclidean cubic lattice with lattice spacing $a$, which we set to unity $(a=1)$. We shall denote a lattice site by the vector $\vec{x}$ and a link between $\vec{x}$ and $\vec{x}+\hat{\mu}(\mu=0,1,2)$ by $(\vec{x}, \hat{\mu})$. Forward and backward difference operators are given by $d_{\mu} f(\vec{x})=f(\vec{x}+\hat{\mu})-f(\vec{x})=$ $\left(S_{\mu}-1\right) f(\vec{x})$ and $\hat{d}_{\mu} f(\vec{x})=f(\vec{x})-f(\vec{x}-\hat{\mu})=(1-$ $\left.S_{\mu}^{-1}\right) f(\vec{x})$, where $S_{\mu} f(\vec{x})=f(\vec{x}+\hat{\mu}), S_{\mu}^{-1} f(\vec{x})=$ $f(\vec{x}-\hat{\mu})$ are the forward and backward shift operators respectively. Summation by parts on the lattice interchanges the forward and backward derivatives: $\sum_{\vec{x}} f(\vec{x}) d_{\mu} g(\vec{x})=-\sum_{\vec{x}} \hat{d}_{\mu} f(\vec{x}) g(\vec{x})$.

The lattice Fourier transformation of the gauge field $A_{\mu}$ is given by

$A_{\mu}(\vec{x})=\int_{\mathcal{B}} \frac{d^{3} p}{(2 \pi)^{3}} e^{-i \vec{p} \cdot \vec{x}} e^{-i p_{\mu} / 2} A_{\mu}(\vec{p}) \quad$.

Due to the phase factor $e^{-i p_{\mu} / 2}, A_{\mu}(\vec{p})$ is antiperiodic if $p_{\mu} \longrightarrow p_{\mu}+2 \pi(2 n+1)$, with $n$ integer. The integration over momenta in eq.(2) is restricted to the Brillouin zone $\mathcal{B}=$ $\left\{p_{\mu} \mid-\pi \leq p_{\mu} \leq \pi, \mu=0,1,2\right\}$. Under parity, which on an Euclidean cubic lattice corresponds to the simultaneous inversion of all three directions,

$A_{\mu}(\vec{x}) \longrightarrow A_{\mu}(-\vec{x}-\hat{\mu})$

and

$A_{\mu}(\vec{p}) \longrightarrow-A_{\mu}(-\vec{p}) \quad$.

The CS action on an Euclidean lattice derived by Frölich and Marchetti [⿰亻] is:

$S=\sum_{\vec{x}} A_{\mu}(\vec{x}) \tilde{K}_{\mu \nu}(\vec{x}-\vec{y}) A_{\nu}(\vec{y}) \quad$,

where $\tilde{K}_{\mu \nu}=K_{\mu \nu}+\hat{K}_{\mu \nu}$, and

$K_{\mu \nu}(\vec{x}-\vec{y})=S_{\mu}^{\vec{y}} \epsilon_{\mu \alpha \nu} d_{\alpha}^{\vec{y}} \delta_{\vec{x}, \vec{y}}$,

$\hat{K}_{\mu \nu}(\vec{x}-\vec{y})=S_{\nu}^{-1, \vec{y}} \epsilon_{\mu \alpha \nu} \hat{d}_{\alpha}^{\hat{y}} \delta_{\vec{x}, \vec{y}}$.

$K$ and $\hat{K}$ are exchanged by summation by parts.
Although both $K$ and $\hat{K}$ define a gauge invariant and parity odd kernel, Bose symmetry 12 requires that only the linear combination $\tilde{K}=$ $K+\hat{K}$ respects this symmetry and thus provides an acceptable definition of the lattice CS action. The operator $\tilde{K}(p)=K(p)+\hat{K}(p)$ has, apart from the zero mode associated with gauge invariance, eigenvalues given by

$\tilde{\lambda}(p)= \pm 2 \sqrt{1+\cos \sum_{\mu=0}^{2} p_{\mu}} \sqrt{3-\sum_{\mu=0}^{2} \cos p_{\mu}}$

and thus $\tilde{\lambda}=0$ whenever $\cos \sum_{\mu=0}^{2} p_{\mu}=-1$, i.e. when $\sum_{\mu=0}^{2} p_{\mu}=(2 n+1) \pi$. The CS action (5) is therefore not integrable.

The properties of $K$ and $\hat{K}$ parallel the ones of the forward and backward derivatives, which in momentum space read $d_{\mu} \rightarrow e^{i p_{\mu} / 2} \hat{p}_{\mu}$ and $\hat{d}_{\mu} \rightarrow e^{-i p_{\mu} / 2} \hat{p}_{\mu}$ with $\hat{p}_{\mu}=2 \sin p_{\mu} / 2$ : they do not have extra zeroes inside the Brillouin zone, but their linear combination $d+\hat{d} \rightarrow 2 \cos \left(p_{\mu} / 2\right) \hat{p}_{\mu}$ has zeros at the border of the Brillouin zone $p_{\mu}= \pm \pi$.

The appearance of the extra zeroes is not due to the specific form of the kernel in (5). In fact, as proven in 10], if the action

$S=\sum_{\vec{x}, \vec{y}} A_{\mu}(\vec{x}) G_{\mu \nu}(\vec{x}-\vec{y}) A_{\nu}(\vec{y})$

i) is local on the lattice;

ii) is gauge invariant: $\hat{d}_{\mu}^{x} G_{\mu \nu}(\vec{x}-\vec{y})=\hat{d}_{\nu}^{y} G_{\mu \nu}(\vec{x}-$ $\vec{y})=0$;

iii) is odd under parity;

(9) is not integrable.

The proof of the theorem is easier in momemtum space. Bose symmetry and parity oddness imply that the kernel $\tilde{G}_{\mu \nu}(\vec{p})=$ $e^{i p_{\mu} / 2} G_{\mu \nu}(\vec{p}) e^{-i p_{\nu} / 2}$ (no sum over $\mu$ and $\nu$ ) is antisymmetric:

$\tilde{G}_{\mu \nu}(\vec{p})=-\tilde{G}_{\nu \mu}(\vec{p})$.

The Poincaré lemma [14] then enable us to rewrite $\tilde{G}_{\mu \nu}(\vec{p})$ in term of a scalar function $f(\vec{p})$ : $\tilde{G}_{\mu \nu}(\vec{p})=\epsilon_{\rho \mu \nu} \hat{p}_{\rho} f(\vec{p})$. Making use of the fact that $G_{\mu \nu}(\vec{p})$ must be a periodic function of the momenta, it is easy to see that for $p_{0}=p_{1}=p_{2}=$ 
$\pm \pi$ one gets

$f( \pm \pi, \pm \pi, \pm \pi)=-f( \pm \pi, \pm \pi, \pm \pi)=0 \quad$.

Since the spectrum of $G_{\mu \nu}(\vec{p})$ is given by $G(\vec{p})=$ $\pm|f(\vec{p})| \sqrt{\sum_{\mu=0}^{2} \hat{p}_{\mu}^{2}}$, eq.(11) implies that the kernel $G(\vec{p})$ exhibits extra zeroes at the edges of the Brillouin zone and is thus not integrable.

Relaxing the assumption iii) one may study the general form of a gauge invariant local action in three dimensions. With the help of the Poincaré lemma [14 it is easy to show that the kernel $\tilde{G}_{\mu \nu}(\vec{p})$ can be divided into the sum of parity even and parity odd terms. Since, due to locality, $\tilde{G}_{\mu \nu}(\vec{p})$ is an analytic function of $\vec{p}$, it may be expanded in Taylor series: all the terms having even power of the momenta are parity even, while the terms with odd power of the momenta are parity odd. The terms with the lowest number of derivatives in this expansion are the CS term defined in [4] and the Maxwell term, whose kernel on the lattice is:

$M_{\mu \nu}=-\square \delta_{\mu \nu}+d_{\mu} \hat{d}_{\nu}=K_{\mu \rho} \hat{K}_{\rho \nu}$,

where $\square=\sum_{\mu=0}^{2} d_{\mu} \hat{d}_{\mu}$ is the Laplacean in three dimensions. Since all the parity odd terms fullfill the assumptions of the theorem they generate extra zeroes in the spectrum. The only gauge invariant way to regularize the CS action is then the addition of a parity even term such as the Maxwell term.

For the Maxwell-CS theory on the lattice the kernel $\Gamma_{\mu \nu}$ may be written as

$\Gamma_{\mu \nu}=\frac{1}{4 e^{2}} M_{\mu \nu}+i k G_{\mu \nu}$.

In (13) $k$ is dimensionless and $e^{2}$ has the dimension of a mass; the Maxwell term is an irrelevant operator and the CS action dominates in the infrared region. The Fourier transform of $\Gamma_{\mu \nu}$, apart from a zero mode due to gauge invariance, has eigenvalues given by

$\lambda_{M C S}(\vec{p})=\frac{1}{2 e^{2}} \sum_{\mu=0}^{2}\left(1-\cos p_{\mu}\right)+i k G(\vec{p})$

and, as it stands, it is free from extra zeroes in the Brillouin zone since the first term in (14), which is the Fourier transform of the Maxwell kernel, is zero only at zero momentum, and at the corners of the Brillouin zone, $p_{\mu}= \pm \pi, \mu=0,1,2$, takes the value $\lambda_{M C S}(\vec{p})=3 / e^{2}$.

Since the CS action is purely immaginary, the addition of the Maxwell term is used also in the continuum theory to provide a proper definition of the functional integral in the partition function of the pure CS theory. The CS limit is reached also there by taking the limit $e^{2} \longrightarrow \infty$ after Gaussian integration.

The regularization of the extra zeros in the CS action by adding a Maxwell term and thereby opening a gap in the fermion spectrum is similar to the mechanism of the Wilson fermion where a gap is opened and the energy does not have secondary minima at the non-zero corners of the Brillouin zone. As in the case of the Wilson fermions [13], the regularization is done by means of an irrelevant operator and the continuum limit $a \longrightarrow 0$ is not changed by this addition. Moreover, as the Wilson action explicitly breaks chiral symmetry, the action obtained after the addition of the Maxwell term is not anymore defined under parity.

\section{Concluding remarks}

In 10 we pointed out a no-go theorem in the lattice regularization of the pure CS theory, if one requires locality, gauge invariance and parity oddness on the lattice. As we already pointed out in the introduction, a doubling phenomenon has been already advocated for bosonic theories on the lattice [6] [7]. In particular the authors of [6] and [7 found a doubling phenomenon for a class of lattice formulations of gravity and in the presence of a gravitational background all matter fields exhibit the same degeneracy as the graviton and the chiral fermion. This doubling phenomenon makes ill defined all matter fields in a gravitational background and it can be removed just in the flat space limit in the case of scalar and gauge bosonic fields. However, in [10] we found a doubling phenomenon for gauge fields completely independent from the metric of the manifold on which the fields live, since the pure CS theory is a topological theory. Our analysis evidences explic- 
itly that doubling phenomena for fields defined on a discrete space-time manifold are a very general phenomena due more to the linearity of the field action in the derivatives than to the curvature of the manifold.

The no-go theorem demonstrated in 10 strongly relies on the definition of the parity transformation for the gauge field $A_{\mu}(\vec{x}) \longrightarrow$ $A_{\mu}(-\vec{x}-\hat{\mu})$. If one could define a new parity on the lattice so that the CS term is still odd but the kernel is integrable, then the new definition of parity should play a role analogous to the Ginsparg-Wilson relation 15] for lattice fermionic theories. For these theories the Ginsparg-Wilson relation hints to a new definition of chiral symmetry on the lattice, a generalization of the usual continuum chiral symmetry that is exactly recovered in the naive continuum limit.

\section{REFERENCES}

1. R. Jackiw and S. Templeton, Phys. Rev. D23 (1981) 2291; J. Schonfeld, Nucl. Phys. B185 (1981) 157; S. Deser, R. Jackiw and S. Templeton, Phys. Rev. Lett. 48 (1982) 957, Ann. Phys. (N.Y.) 140 (1982) 372.

2. E. Witten, Comm. Math. Phys. 121 (1989) 351, Int. Jour. Mod. Phys. A6 (1991) 2775.

3. For a review see: A Zee, Prog. Theor. Phys. Supp. 107 (1992) 77; M.C. Diamantini P. Sodano and C.A. Trugenberger, Nucl. Phys. B 448, (1995) 505, Nucl. Phys. B 474 (1996) 641

4. J. Frölich and P.-A. Marchetti, Comm. Math. Phys. 121 (1989) 177.

5. J. Kogut and L. Susskind, Phys. Rev. D11 (1975) 395; L. Susskind, Phys. Rev. D16 (1977) 3031; K. G. Wilson, "New Phenomena in Subnuclear Physics" (Erice, 1975), ed. A. Zichichi (New York, Plenum, 1975).

6. P. Menotti and A. Pelissetto, Ann. of Phys. (NY) 170 (1986) 287.

7. M. Caselle, A. D'Adda and L. Magnea, Phys. Lett. 192 (1987) 411.

8. D. Eliezer and G. W. Semenoff, Ann. of Phys. 217 (1992) 66; D. Eliezer and G. W. Semenoff, Phys. Lett. B286 (1992) 118.

9. R. Kantor and L. Susskind, Nucl. Phys. B366 (1993) 533;D. Eliezer and G. W. Semenoff,
Phys. Lett. B266, (1991) 375; D. H. Adams, Phys. Rev. Lett. 78 (1997) 4155.

10. F. Berruto, M. C. Diamantini and P. Sodano, Phys. Lett. B487 (2000) 366.

11. M. Lüscher, Nucl. Phys. B326 (1989) 557; V.F. Müller, Z. Phys. C 47 (1990) 301; M.C. Diamantini, P. Sodano and C.A. Trugenberger, Phys. Rev. Lett. 71 (1993) 1969, Phys. Rev. Lett. 75 (1995) 3517.

12. L. H. Karsten and J. Smit, Nucl. Phys. B183 (1981) 103.

13. J. Kogut and K.G. Wilson, Phys. Rev. C12 (1974) 75 .

14. M. Lüscher, Nucl. Phys. B538 (1999) 515.

15. P. H. Ginsparg and K. G. Wilson, Phys. Rev. D25 (1982) 2649. 